\title{
Effect of Hops (Humulus Lupulus) Supplementation on Growth Performance of Broiler Chickens
}

\author{
Fasina Y0* and Akinola 00 \\ Department of Animal Sciences, USA
}

*Corresponding author: Fasina YO, Department of Animal Sciences, Greensboro, NC 27411 USA

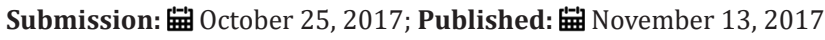

\begin{abstract}
The hop plant contains flavonoids, bitter acids and essential oils that confer antibacterial properties. An experiment was conducted to evaluate the possibility of supplementing broiler chicken diets with hop pellets. Accordingly, growth performance response of broiler chickens given hopssupplemented diets was evaluated in a 49-day floor-pen trial. Day-old (320) male broiler chicks were commercially obtained and randomly assigned to 4 treatments. Treatment 1 (CX) consisted of chicks fed unmedicated corn-soybean meal (SBM) diet without hops pellets added. Treatment 2 (MX) consisted of chicks fed corn-SBM basal into which bacitracin methylene disalicylate (BMD) was added at $0.055 \mathrm{~g} / \mathrm{kg}$. Treatment 3 (HL) consisted of chicks fed corn-SBM basal into which hops pellets were added at $1.0 \%$ level. Treatment 4 (HH) consisted of chicks fed corn-SBM basal into which hops pellets were added at $2.0 \%$ level. On d 21, 42, and 49, body weight, body weight gain, and feed conversion ratio (FCR) were evaluated. Transient benefits of hops supplementation in form of higher body weight gain $(\mathrm{P}<0.05)$ compared to other treatments were observed but were not sustained. The FCR of birds in the hops-supplemented treatments (HL and HH) were similar $(\mathrm{P}>0.05)$ to those of birds in the BMD antibiotic-supplemented treatment (MX) throughout the study. It was concluded that supplementation of hops pellets into broiler diets at $1 \%$ or $2 \%$ level of the diet had no detrimental effect on broiler growth performance. Therefore, future studies should be conducted to evaluate the efficacy of dietary hops in mitigating the colonization of poultry intestine by economically important zoonotic and/or disease pathogens .
\end{abstract}

Keywords: Hops pellet; Bacitracin methylene disalicylate; Broiler chickens; Growth performance

Abbreviations: SBM: Soybean Meal; BMD: Bacitracin Methylene Disalicylate; BWG: Body Weight Gain; FCR: Feed Conversion Ratio; GLM: General Linear Models

\section{Introduction}

The movement towards prohibiting the use of antibiotics in animal feed has become stronger in recent years due to the emergence of antibiotic resistant bacterial strains. Resistant strains of zoonotic pathogens such as Salmonella spp., Campylobacter spp., Escherichia coli, and Clostridium perfringens have been isolated from the intestines of commercial broiler chickens [1-4]. Ongoing efforts to replace antibiotics with alternative biogenics such as direct-fed microbials, yeast-based products, organic acids, essential oils, antimicrobial peptides, acidifiers, and plant extracts in poultry diets have met limited success because, none of these alternatives is able to solely replace antibiotics [5-7]. This warrants continuing research efforts to identify novel effective alternatives, and also perhaps formulate superior combination(s) of these products.

Hops (Humulus lupulus L.) is a plant that contain various compounds that have been determined as beneficial for therapeutic use in human medicine. In particular, it contains various flavonoids, bitter acids (comprising of humulones ( $\alpha$-acids), lupulones ( $\beta$-acids), isohumulones (iso-a-acids)), and essential oils that are responsible for its antibacterial, antioxidant, and digestion accelerating properties [8]. In addition, the hop plant is recognized as generally safe for use in brewing and in other human foods. It has been reported that hop extracts containing $\beta$-acids and xanthohumol inhibited the growth of Gram-positive bacteria in vitro with minimum inhibitory values of 6.3 and $12.5 \mathrm{ppm}$, respectively [9]. Information is scanty regarding the possibility of using hops (and products) as alternatives to antibiotics in live poultry. Siragusa et al. [10] reported that untreated chickens challenged with $C$. perfringens had higher $(\mathrm{P}<0.05)$ C. perfringens counts in the jejunum and ceca $(\log 106.20 \mathrm{cfu} / \mathrm{g})$, compared to their counterparts treated with administration of $62.5,125$, or $250 \mathrm{ppm}$ lupulone extract in tap water $(\log 102.00 \mathrm{cfu} / \mathrm{g})$. Similarly, Tillman et al. [11] observed that administration of lupulone at $125 \mathrm{mg} \mathrm{L}^{-1}$ significantly decreased the $C$. perfringens subgroup $16 \mathrm{~S}$ rRNA gene Cluster I (which contains several pathogenic species) in the midgut and cecum of broiler chickens challenged with $C$. perfringens.

There have been no attempt to include hops (or its products) in the diet of poultry. Dietary inclusion of hops in broiler diets may allow greater realization of its potential antibacterial, antioxidant, and digestion accelerating properties. To promote the inclusion of hops in poultry diets, it will be necessary to first determine the 
optimum level of inclusion with respect to feed intake, palatability, and growth performance. The objective of this study was to evaluate the growth performance response of broiler chickens given hopssupplemented diets. Growth performance indices such as body weight, body weight gain, feed intake, and feed conversion ratio were evaluated on days 21,42 , and 49 of experiment.

\section{Materials and Methods}

All the procedures used in this study were approved by the Auburn University Institutional Animal Care and Use Committee.

\section{Experimental animals and treatments}

Day-old male broiler chicks (320; Cobb x Cobb) were obtained from a commercial hatchery and utilized in a 49-day floor-pen trial that simulated industry settings. Chicks were weighed, wing-banded, and randomly assigned to four dietary treatments. Treatment 1 (CX) consisted of chicks fed unmedicated corn- soybean meal (SBM) diet without hops pellets added. Treatment 2 (MX) consisted of chicks fed corn-SBM basal into which bacitracin methylene disalicylate (BMD) was added at $0.055 \mathrm{~g} / \mathrm{kg}$. Treatment 3 (HL) consisted of chicks fed corn-SBM basal into which hops pellets were added at $1.0 \%$ level. Treatment 4 (HH) consisted of chicks fed corn-SBM basal into which hops pellets were added at $2.0 \%$ level.

The Cascade hops pellets used in this study were obtained from S.S. Steiner, Inc. (New York, NY) and were certified to contain 4.5 to $7 \%$ alpha -acid (humulones) and 4.5 to $7 \%$ beta-acid (lupulone). The hops pellets were crumbled prior to being included into HL and $\mathrm{HH}$ diets. Experimental diets were formulated to meet the recommendations of the National Research Council [12], and their nutrient compositions are presented in Tables 1-3. Starter diets were fed to chicks from day-old to 3 weeks as a crumble after steampelleting. Grower diets were fed as whole pellets from 3 to 6 weeks of age, and finisher diets were fed as whole pellets during week 7 .

Table 1: Composition of experimental starter diets (\% "as is").

\begin{tabular}{|c|c|c|c|c|}
\hline Ingredient & CX diet $^{1}$ & MX Diet ${ }^{1}$ & HL Diet $^{1}$ & HH Diet $^{1}$ \\
\hline Corn & 55.04 & 55.04 & 54.04 & 53.04 \\
\hline Soybean meal & 36.75 & 36.75 & 36.75 & 36.75 \\
\hline Poultry oil & 3.7 & 3.7 & 3.7 & 3.7 \\
\hline DL-Methionine & 0.19 & 0.19 & 0.19 & 0.19 \\
\hline Limestone & 1.68 & 1.68 & 1.68 & 1.68 \\
\hline Dicalcium Phosphate & 1.74 & 1.74 & 1.74 & 1.74 \\
\hline Vitamin Premix ${ }^{2}$ & 0.25 & 0.25 & 0.25 & 0.25 \\
\hline Mineral Premix ${ }^{3}$ & 0.25 & 0.25 & 0.25 & 0.25 \\
\hline Salt & 0.4 & 0.4 & 0.4 & 0.4 \\
\hline Bacitracin (Antibiotic, g/kg) & --- & 0.055 & --- & --- \\
\hline Hops Pellet (\%) & ---- & --- & 1 & 2 \\
\hline \multicolumn{5}{|c|}{ Analyzed nutrient composition ${ }^{4}$} \\
\hline $\begin{array}{l}\text { Metabolizable energy (Kcal/ } \\
\mathrm{kg})\end{array}$ & 3256 & 3144 & 3106 & 3115 \\
\hline Crude protein, $\%$ & 22.7 & 22.81 & 22.41 & 22.01 \\
\hline Crude fat, $\%$ & 6.29 & 5.73 & 5.78 & 5.61 \\
\hline Crude fiber, $\%$ & 2.2 & 2.6 & 3 & 3.3 \\
\hline \multicolumn{5}{|c|}{ Calculated nutrient composition } \\
\hline Total Sulfur Amino Acids, \% & 0.89 & 0.89 & 0.89 & 0.89 \\
\hline Lysine, $\%$ & 1.24 & 1.24 & 1.24 & 1.24 \\
\hline Calcium, \% & 1.11 & 1.11 & 1.11 & 1.11 \\
\hline Available phosphorus, \% & 0.46 & 0.46 & 0.46 & 0.46 \\
\hline
\end{tabular}

${ }^{1}$ Diets used in this study included i) the Control (CX) diet comprising of corn-soybean meal (SBM) basal without bacitracin methylene disalicylate (BMD) or hops pellet added, ii) the medicated diet (MX) comprising of corn-SBM basal into which BMD was added at $0.055 \mathrm{~g} / \mathrm{kg}$, iii) the low hops (HL) diet comprising of corn-SBM basal into which hops pellet was added at $1.0 \%$ level, and iv) the high hops $(\mathrm{HH})$ diet comprising of corn-SBM basal into which hops pellet was added at $2.0 \%$ level.

${ }^{2}$ Vitamin Premix, supplied per kilogram of diet: vitamin A (retinyl acetate), 7356IU; vitamin D3 (cholecalciferol), 2,205ICU; vitamin E, (8 IU); vitamin B12 (cyanocobalamin), 0.2mg; riboflavin, 5.5mg; niacin, 36mg; D-pantothenic acid, 13mg; choline, 501mg; vitamin K (menadione sodium bisulfate), $2 \mathrm{mg}$; folic acid, $0.5 \mathrm{mg}$; vitamin B6 (pyridoxine), 2.2; vitamin B1 (thiamin), 1.0mg; D-biotin, 0.5mg; and ethoxyquin, $0.1 \mathrm{mg}$.

${ }^{3}$ Mineral Premix, supplied per kilogram of diet: manganese, $65 \mathrm{mg}$; zinc, $55 \mathrm{mg}$; iron, $55 \mathrm{mg}$; copper, $6 \mathrm{mg}$; iodine, $1 \mathrm{mg}$; and selenium, $0.3 \mathrm{mg}$.

${ }^{4}$ Diets were analyzed by Eurofins Scientific Inc., Nutrition Analysis Center, 2200 Rittenhouse St., Suite 150, Des Moines, IA 50321. 
Table 2: Composition of experimental grower diets (\% "as is").

\begin{tabular}{|c|c|c|c|c|}
\hline Ingredient & CX $\operatorname{diet}^{1}$ & MX Diet ${ }^{1}$ & HL Diet $^{1}$ & HH Diet $^{1}$ \\
\hline Corn & 65.36 & 65.36 & 64.36 & 63.36 \\
\hline Soybean meal & 29.48 & 29.48 & 29.48 & 29.48 \\
\hline Poultry oil & 1.54 & 1.54 & 1.54 & 1.54 \\
\hline DL-Methionine & 0.65 & 0.65 & 0.65 & 0.65 \\
\hline Limestone & 1.43 & 1.43 & 1.43 & 1.43 \\
\hline Dicalcium Phosphate & 1.32 & 1.32 & 1.32 & 1.32 \\
\hline Vitamin Premix ${ }^{2}$ & 0.25 & 0.25 & 0.25 & 0.25 \\
\hline Mineral Premix ${ }^{3}$ & 0.25 & 0.25 & 0.25 & 0.25 \\
\hline Salt & 0.3 & 0.3 & 0.3 & 0.3 \\
\hline Bacitracin (Antibiotic, g/kg) & --- & 0.055 & --- & --- \\
\hline Hops Pellet (\%) & --- & --- & 1 & 2 \\
\hline \multicolumn{5}{|c|}{ Calculated nutrient composition } \\
\hline Metabolizable energy (Kcal/kg) & 3058 & 3058 & 3024 & 2990 \\
\hline Crude protein, $\%$ & 20.12 & 20.12 & 20.05 & 20 \\
\hline Crude fat, $\%$ & 4.33 & 4.33 & 4.29 & 4.26 \\
\hline Crude fiber, $\%$ & 2.76 & 2.76 & 2.73 & 2.71 \\
\hline Total Sulfur Amino Acids, $\%$ & 0.72 & 0.72 & 0.72 & 0.72 \\
\hline Lysine, $\%$ & 1.03 & 1.03 & 1.03 & 1.03 \\
\hline Calcium, \% & 0.89 & 0.89 & 0.89 & 0.89 \\
\hline Available phosphorus, $\%$ & 0.36 & 0.36 & 0.36 & 0.36 \\
\hline
\end{tabular}

${ }^{1}$ Diets used in this study included i) the Control (CX) diet comprising of corn-soybean meal (SBM) basal without bacitracin methylene disalicylate (BMD) or hops pellet added, ii) the medicated diet (MX) comprising of corn-SBM basal into which BMD was added at $0.055 \mathrm{~g} / \mathrm{kg}$, iii) the low hops (HL) diet comprising of corn-SBM basal into which hops pellet was added at $1.0 \%$ level, and iv) the high hops $(\mathrm{HH})$ diet comprising of corn-SBM basal into which hops pellet was added at $2.0 \%$ level.

${ }^{2}$ Vitamin Premix, supplied per kilogram of diet: vitamin A (retinyl acetate), 7356IU; vitaminD3 (cholecalciferol), 2,205ICU; vitamin E, (8 IU); vitamin B12 (cyanocobalamin), 0.2mg; riboflavin, 5.5mg; niacin, 36mg; D-pantothenic acid, $13 \mathrm{mg}$; choline, $501 \mathrm{mg}$; vitamin K (menadione sodium bisulfate), 2mg; folic acid, 0.5mg; vitamin B6 (pyridoxine), 2.2; vitamin B1 (thiamin), $1.0 \mathrm{mg}$; D-biotin, $0.5 \mathrm{mg}$; and ethoxyquin, $0.13 \mathrm{mg}$.

${ }^{3}$ Mineral Premix, supplied per kilogram of diet: manganese, 65mg; zinc, 55mg; iron, 55mg; copper, 6mg; iodine, $1 \mathrm{mg}$; and selenium, $0.3 \mathrm{mg}$. 
Table 3: Composition of experimental finisher diets (\% "as is").

\begin{tabular}{|c|c|c|c|c|}
\hline Ingredient & CX $\operatorname{diet}^{1}$ & MX Diet ${ }^{1}$ & HL Diet $^{1}$ & HH Diet $^{1}$ \\
\hline Corn & 66.45 & 66.45 & 65.45 & 64.45 \\
\hline Soybean meal & 26.8 & 26.8 & 26.8 & 26.8 \\
\hline Poultry oil & 3.49 & 3.49 & 3.49 & 3.49 \\
\hline DL-Methionine & 0.19 & 0.19 & 0.19 & 0.19 \\
\hline Limestone & 1.02 & 1.02 & 1.02 & 1.02 \\
\hline Dicalcium Phosphate & 1.1 & 1.1 & 1.1 & 1.1 \\
\hline Vitamin Premix ${ }^{2}$ & 0.25 & 0.25 & 0.25 & 0.25 \\
\hline Mineral Premix ${ }^{3}$ & 0.25 & 0.25 & 0.25 & 0.25 \\
\hline Salt & 0.47 & 0.47 & 0.47 & 0.47 \\
\hline Bacitracin (Antibiotic, g/kg) & --- & 0.055 & --- & --- \\
\hline Hops Pellet (\%) & --- & ---- & 1 & 2 \\
\hline \multicolumn{5}{|c|}{ Calculated nutrient composition } \\
\hline $\begin{array}{l}\text { Metabolizable energy (Kcal/ } \\
\text { kg) }\end{array}$ & 3203 & 3203 & 3169 & 3135 \\
\hline Crude protein, $\%$ & 17.97 & 17.97 & 17.89 & 17.82 \\
\hline Crude fat, $\%$ & 6.31 & 6.31 & 6.27 & 6.23 \\
\hline Crude fiber, \% & 2.68 & 2.68 & 2.66 & 2.63 \\
\hline Total Sulfur Amino Acids, $\%$ & 0.8 & 0.8 & 0.8 & 0.8 \\
\hline Lysine, $\%$ & 0.96 & 0.96 & 0.96 & 0.96 \\
\hline Calcium, \% & 0.69 & 0.69 & 0.69 & 0.69 \\
\hline Available phosphorus, \% & 0.32 & 0.32 & 0.32 & 0.32 \\
\hline
\end{tabular}

${ }^{1}$ Diets used in this study included i) the Control (CX) diet comprising of corn-soybean meal (SBM) basal without bacitracin methylene disalicylate (BMD) or hops pellet added, ii) the medicated diet (MX) comprising of corn-SBM basal into which BMD was added at $0.055 \mathrm{~g} / \mathrm{kg}$, iii) the low hops (HL) diet comprising of corn-SBM basal into which hops pellet was added at $1.0 \%$ level, and iv) the high hops (HH) diet comprising of corn-SBM basal into which hops pellet was added at $2.0 \%$ level.

${ }^{2}$ Vitamin Premix, supplied per kilogram of diet: vitamin A (retinyl acetate), 7356IU; vitamin D3 (cholecalciferol), 2,205ICU; vitamin E, (8IU); vitamin B12 (cyanocobalamin), 0.2mg; riboflavin, 5.5mg; niacin, 36mg; D-pantothenic acid, 13mg; choline, 501mg; vitamin K (menadione sodium bisulfate), 2mg; folic acid, 0.5mg; vitamin B6 (pyridoxine), 2.2; vitamin B1 (thiamin), 1.0mg; D-biotin, 0.5mg; and ethoxyquin, $0.13 \mathrm{mg}$.

${ }^{3}$ Mineral Premix, supplied per kilogram of diet: manganese, 65mg; zinc, 55mg; iron, 55mg; copper, 6mg; iodine, 1mg; and selenium, $0.3 \mathrm{mg}$.

Experimental chicks were kept in pens inside a house with cross ventilation and temperature control. Each treatment had 4 replicate pens, with each pen containing 20 chicks placed on fresh pine shaving litter. Chicks were provided with continuous lighting commensurate with day (1,400 to 2,800 lx) and night (4 to $17 \mathrm{~lx}$ ) intensities. Chicks were allowed ad -libitum access to feed and water throughout the experiment. Duration of experiment was 49 days (i.e. 7 weeks).

\section{Assessment of growth performance and statistical analysis}

On days 21, 42, and 49 of experiment, body weight, body weight gain (BWG), feed intake, and feed conversion ratio (FCR) were calculated for the evaluation of broiler growth performance. Mortality was also recorded on daily basis. Data collected were subjected to one-way ANOVA using the General Linear Models (GLM) procedure of SAS [13]. Significant differences among means were determined using the Duncan option of the GLM procedure as a post hoc test, and statements of statistical significance were based upon $\mathrm{P}<0.05$. Data are presented as means \pm SEM.

\section{Results and Discussion}

An experiment was conducted to determine if dietary supplementation of hops at 1 or $2 \%$ level of the diet will adversely affect body weight, body weight gain, and FCR of broiler chickens. Results obtained from this study are presented in Tables 4-7. On day 21 , there were no differences $(\mathrm{P}>0.05)$ in body weight, body weight gain, and feed conversion efficiency (FCR) of chicks in all treatments (Table 4). On the other hand, there were differences in feed intake. Specifically, chicks in the HH treatment consumed a significantly higher $(\mathrm{P}<0.05)$ quantity of feed $(2.03 \mathrm{~kg} /$ chick $)$ compared to chicks in CX $(1.23 \mathrm{~kg} /$ chick $), \mathrm{MX}(1.29 \mathrm{~kg} /$ chick$)$, and HL(1.29kg/chick). The higher feed intake in $\mathrm{HH}$ translated to numerically higher body weight and body weight gain in the chicks, but these did not culminate in a significantly superior FCR compared to other treatments. It was also important to include 
the BMD antibiotic-supplemented MX treatment in this study for comparison with hops as its candidate replacement. Chicks ( $\mathrm{P}>0.05$ ) body weight, Body weight gain, and FCR to chicks in the given the hops- supplemented diets (HL and $\mathrm{HH}$ ) had comparable

Table 4: Effect of Hops supplementation on growth performance during starter phase (Day 1 to 21).

\begin{tabular}{|c|c|c|c|c|}
\hline Treatments $^{1}$ & Body Weight $(\mathrm{kg} / \mathrm{bird})^{2}$ & Body Weight Gain (kg/bird) ${ }^{2}$ & Feed Intake (kg/bird) & Feed Conversion Ratio (kg:kg) \\
\hline $\mathrm{CX}$ & 0.837 & 0.792 & $1.29^{\mathrm{b}}$ & 1.41 \\
\hline MX & 0.82 & 0.777 & $1.29^{\mathrm{b}}$ & 1.41 \\
\hline HL & 0.82 & 0.775 & $1.23^{\mathrm{b}}$ & 1.43 \\
\hline $\mathrm{HH}$ & 0.852 & 0.81 & $2.03^{\mathrm{a}}$ & 1.38 \\
\hline SEM & 0.025 & 0.024 & 0.101 & 0.043 \\
\hline P-value & 0.7649 & 0.7162 & 0.0003 & 0.8408 \\
\hline
\end{tabular}

${ }^{a, b}$ Mean values bearing different superscript letters within a column are significantly different $(\mathrm{P}<0.05)$.

${ }^{1}$ Treatment 1 (CX) consisted of chicks fed unmedicated corn-soybean meal (SBM) diet without hops pellets added. Treatment 2 (MX) consisted of chicks fed corn-SBM basal into which bacitracin methylene disalicylate (BMD) was added at $0.055 \mathrm{~g} / \mathrm{kg}$. Treatment 3 (HL) consisted of chicks fed corn-SBM basal into which hops pellets were added at $1.0 \%$ level. Treatment 4 (HH) consisted of chicks fed corn-SBM basal into which hops pellets were added at $2.0 \%$ level.

${ }^{2}$ Values are based only on weight of live birds.

${ }^{3}$ Feed conversion ratio calculated as feed-to-gain ratio and adjusted for mortality by including the gains of dead birds in the calculations.

Table 5: Effect of Hops supplementation on growth performance during grower phase (Day 22 to 42).

\begin{tabular}{|c|c|c|c|c|}
\hline Treatments $^{1}$ & Body Weight $(\mathrm{kg} / \mathrm{bird})^{2}$ & Body Weight Gain (kg/bird) ${ }^{2}$ & Feed Intake (kg/bird) & Feed Conversion Ratio (kg:kg) ${ }^{3}$ \\
\hline CX & 2.69 & 1.85 & 3.73 & 1.78 \\
\hline MX & 2.72 & 1.90 & 3.71 & 1.72 \\
\hline HL & 2.69 & 1.87 & 3.49 & 1.67 \\
\hline $\mathrm{HH}$ & 2.73 & 1.87 & 3.63 & 1.74 \\
\hline SEM & 0.082 & 0.070 & 0.097 & 0.060 \\
\hline P-value & 0.9795 & 0.9613 & 0.3394 & 0.6410 \\
\hline
\end{tabular}

${ }^{1}$ Treatment $1(\mathrm{CX})$ consisted of chicks fed unmedicated corn-soybean meal (SBM) diet without hops pellets added. Treatment 2 (MX) consisted of chicks fed corn-SBM basal into which bacitracin methylene disalicylate (BMD) was added at $0.055 \mathrm{~g} / \mathrm{kg}$. Treatment 3 (HL) consisted of chicks fed corn-SBM basal into which hops pellets were added at $1.0 \%$ level. Treatment 4 (HH) consisted of chicks fed corn-SBM basal into which hops pellets were added at $2.0 \%$ level.

${ }^{2}$ Values are based only on weight of live birds.

${ }^{3}$ Feed conversion ratio calculated as feed-to-gain ratio and adjusted for mortality by including the gains of dead birds in the calculations.

Table 6: Effect of Hops supplementation on growth performance during finisher phase (Day 42 to 49).

\begin{tabular}{|c|c|c|c|c|}
\hline Treatments $^{1}$ & Bodyweight (kg/bird $)^{2}$ & Body Weight Gain (kg/bird) ${ }^{2}$ & Feed Intake (kg/bird) & Feed Conversion Ratio (kg:kg) \\
\hline $\mathrm{CX}$ & 3.32 & $0.62^{\mathrm{ab}}$ & 1.46 & 2.14 \\
\hline MX & 3.25 & $0.53^{\mathrm{b}}$ & 1.32 & 2.33 \\
\hline HL & 3.29 & $0.60^{\mathrm{ab}}$ & 1.39 & 2.09 \\
\hline $\mathrm{HH}$ & 3.44 & $0.72^{\mathrm{a}}$ & 1.52 & 2.07 \\
\hline SEM & 0.099 & 0.046 & 0.091 & 0.131 \\
\hline P-value & 0.5540 & 0.0590 & 0.4353 & 0.5043 \\
\hline
\end{tabular}

${ }^{a, b}$ Mean values bearing different superscript letters within a column are significantly different $(\mathrm{P}<0.05)$.

${ }^{1}$ Treatment 1 (CX) consisted of chicks fed unmedicated corn-soybean meal (SBM) diet without hops pellets added. Treatment 2 (MX) consisted of chicks fed corn-SBM basal into which bacitracin methylene disalicylate (BMD) was added at $0.055 \mathrm{~g} / \mathrm{kg}$. Treatment 3 (HL) consisted of chicks fed corn-SBM basal into which hops pellets were added at $1.0 \%$ level. Treatment 4 (HH) consisted of chicks fed corn-SBM basal into which hops pellets were added at $2.0 \%$ level.

${ }^{2}$ Values are based only on weight of live birds.

${ }^{3}$ Feed conversion ratio calculated as feed-to-gain ratio and adjusted for mortality by including the gains of dead birds in the calculations. 
Table 7: Effect of Hops supplementation on cumulative growth performance (Day 1 to 49).

\begin{tabular}{|c|c|c|c|}
\hline Treatments $^{\mathbf{1}}$ & ${\text { Body Weight } \mathbf{( k g} / \mathbf{b i r d})^{\mathbf{2}}}$ & Feed Intake (kg/bird) & ${\text { Feed Conversion Ratio (kg: } \mathbf{k g})^{\mathbf{3}}}$ \\
\hline CX & 3.32 & $6.48^{\mathrm{b}}$ & 3.32 \\
\hline MX & 3.25 & $6.32^{\mathrm{b}}$ & 3.25 \\
\hline HL & 3.29 & $6.12^{\mathrm{b}}$ & 3.29 \\
\hline HH & 3.44 & $7.19^{\mathrm{a}}$ & 3.44 \\
\hline SEM & 0.099 & 0.187 & 0.038 \\
\hline P-value & 0.5540 & 0.0085 & 0.4187 \\
\hline
\end{tabular}

a,b Mean values bearing different superscript letters within a column are significantly different $(\mathrm{P}<0.05)$.

${ }^{1}$ Treatment 1 (CX) consisted of chicks fed unmedicated corn-soybean meal (SBM) diet without hops pellets added. Treatment 2 (MX) consisted of chicks fed corn-SBM basal into which bacitracin methylene disalicylate (BMD) was added at $0.055 \mathrm{~g} / \mathrm{kg}$. Treatment 3 (HL) consisted of chicks fed corn-SBM basal into which hops pellets were added at $1.0 \%$ level. Treatment 4 (HH) consisted of chicks fed corn-SBM basal into which hops pellets were added at $2.0 \%$ level.

${ }^{2}$ Values are based only on weight of live birds.

${ }^{3}$ Feed conversion ratio calculated as feed-to-gain ratio and adjusted for mortality by including the gains of dead birds in the calculations.

Between d 22 and 42, no differences were observed $(\mathrm{P}>0.05)$ among treatments for any of the parameters evaluated (Table 5). However, on $d 49$, there were differences among treatments $(\mathrm{P}<0.05$ ) in weight gain from d 42 to 49 (Table 6). Birds in $\mathrm{HH}$ treatment had a significantly higher weight gain $(0.72 \mathrm{~kg} / \mathrm{bird})$ compared to those in MX $(0.53 \mathrm{~kg} / \mathrm{bird})$. Weight gain values were similar for CX, MX, and HL. Looking at the overall bird performance from d 1 to 49, there were no differences in body weight and FCR among treatments $(\mathrm{P}>0.05)$. However, feed intake was higher for $\mathrm{HH}$ treatment $(\mathrm{P}<0.05)$ compared to other treatments. Total mortality in this study was $3.13 \%$, and this is below $4.4 \%$ reported by National Chicken Council in 2017 as typical for U.S. broiler flocks [14]. In addition, mortality levels were similar $(\mathrm{P}>0.05)$ among treatments (Figure 1).

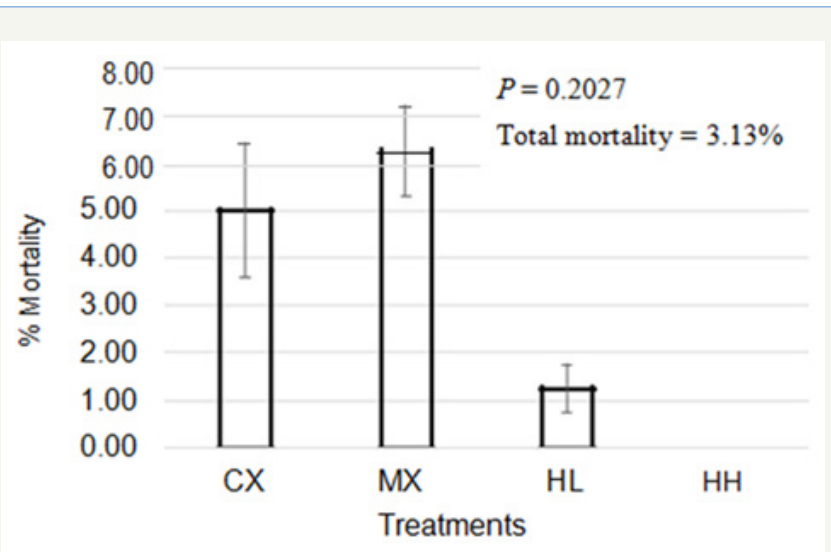

Figure 1: Effect of Hops supplementation on mortality of broiler chickens.

Treatment 1 (CX) consisted of chicks fed unmedicated corn-soybean meal (SBM) diet without hops pellets added. Treatment 2 (MX) consisted of chicks fed corn-SBM basal into which bacitracin methylene disalicylate (BMD) was added at $0.055 \mathrm{~g} / \mathrm{kg}$. Treatment $3(\mathrm{HL})$ consisted of chicks fed corn-SBM basal into which hops pellets were added at $1.0 \%$ level. Treatment 4 (HH) consisted of chicks fed cornSBM basal into which hops pellets were added at $2.0 \%$ level.
Although hops are known to contain bitter acids, this did not seem to affect palatability of the diets in this study because, birds in HL and HH treatments had similar FCR $(\mathrm{P}>0.05)$ to hop-free treatments (CX and MX).

\section{Conclusion}

In conclusion, supplementation of hops pellets into broiler diets at $1 \%$ or $2 \%$ level of the diet had no detrimental effect on broiler growth performance under the conditions of this experiment. Therefore, future studies should be conducted to evaluate the efficacy of dietary hops in mitigating the colonization of poultry intestine by economically important zoonotic and/or disease pathogens such as Salmonella spp., Campylobacter spp., Escherichia coli, and Clostridium perfringens.

\section{Acknowledgement}

This research was funded, in part, by the NIFA through the Agricultural Research Program at North Carolina Agricultural and Technical State University (Evans-Allen Program, project number NC.X-305-5-17-120-1).

\section{References}

1. Slavic D, Boerlin P, Fabri M, Klotins KC, Zoethout JK et al. (2011) Antimicrobial susceptibility of Clostridium perfringens isolates of bovine, chicken, porcine, and turkey origin from Ontario. Can J Vet Res 75(2): 89-97.

2. de Jong A, Thomas V, Simjee S, Godinho K, Schiessl B, et al. (2012) PanEuropean monitoring of susceptibility to human-use antimicrobial agents in enteric bacteria isolated from healthy food-producing animals. 2012. J Antimicrob Chemother 67(3): 638-651.

3. Feye KM, Anderson KL, Scott MF, McIntyre DR, Carlson SA (2016) Inhibition of the virulence, antibiotic resistance, and fecal shedding of multiple antibiotic-resistant Salmonella Typhimurium in broilers fed

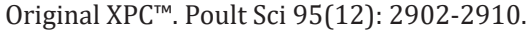

4. Nhung NT, Chansiripornchai N, Carrique-MJJ (2017) Antimicrobial Resistance in Bacterial Poultry Pathogens: A Review Front Vet Sci 4: 126.

5. Seal BS, Lillehoj HS, Donovan DM, Gay CG (2013) Alternatives to antibiotics: a symposium on the challenges and solutions for animal production. Anim Health Res Rev 14(1): 78-87. 
6. Allen HK, Trachsel J, Looft T, Casey TA (2014) Finding alternatives to antibiotics. Ann NY Acad Sci 1323(2014): 91-100.

7. Wang J, Han M, Zhang G, Qiao S, Li D, et al. (2016) The Signal Pathway of Antibiotic Alternatives on Intestinal Microbiota and Immune Function. Curr Protein Pept Sci 17(8): 785-796.

8. Masek A, Chrzescijanska E, Kosmalska A, Zaborski M (2014) Characteristics of compounds in hops using cyclic voltammetry, UV-VIS, FTIR and GC-MS analysis. Food Chem 156: 353-361.

9. Kramer B, Thielmann J, Hickisch A, Muranyi P, Wunderlich J, et al. (2014) Antimicrobial activity of hop extracts against foodborne pathogens for meat applications. J Appl Micro 118(3): 648-657.

10. Siragusa GR, Haas GJ, Matthews PD, Smith RJ, Buhr RJ, et al. (2008)
Antimicrobial activity of lupulone against Clostridium perfringens in the chicken intestinal tract jejunum and caecum. J Antimicrob Chemother 61(4): 853-858.

11. Tillman GE, Haas GJ, Wise MG, Oakley B, Smith MA, et al. (2011) Chicken intestine microbiota following the administration of lupulone, a hopbasedantimicrobial. FEMS Microbiol Ecol 77(2): 395-403.

12. National Research Council (1994) Nutrient Requirements of Poultry. ( $9^{\text {th }}$ rev edn.) Natl Acad Press, Washington, DC,USA.

13. SAS Institute (2004) SAS/STAT User's Guide. Version 9.1 for Windows. SAS Inst Inc, Cary, NC.

14. National Chicken Council (2017) US broiler performance. 